\title{
Meta
}

Journal des traducteurs

Translators' Journal

\section{Janzen, T. (ed.) (2005) : Topics in Signed Language Interpreting: Theory and Practice, Philadelphia, John Benjamins, $362 \mathrm{p}$.}

\section{Danielle-Claude Bélanger}

Volume 54, numéro 1, janvier 2009

URI : https://id.erudit.org/iderudit/029801ar

DOI : https://doi.org/10.7202/029801ar

Aller au sommaire du numéro

Éditeur(s)

Les Presses de l'Université de Montréal

ISSN

0026-0452 (imprimé)

1492-1421 (numérique)

Découvrir la revue

Citer ce compte rendu

Bélanger, D.-C. (2009). Compte rendu de [Janzen, T. (ed.) (2005) : Topics in Signed Language Interpreting : Theory and Practice, Philadelphia, John Benjamins, 362 p.] Meta, 54(1), 170-174. https://doi.org/10.7202/029801ar d'utilisation que vous pouvez consulter en ligne. 
ou pour d'autres combinaisons linguistiques que l'anglais et le français, ouvrant ainsi de nouvelles perspectives de recherche et constituant un outil utile pour les traducteurs soucieux de mieux comprendre les techniques narratives et d'y adapter leurs stratégies de traduction.

Serge Marcoux

Université de Montréal, Montréal, Canada

\section{NOTE}

1. Gérard de Cortanze dans le Magazine Littéraire (1993) en dira: «Elle ôte les aspérités. Elle police plus qu'elle ne polit le texte, fait de ce parc anglais un jardin à la française» (traduction de Charlotte Brosseaux).

\section{RÉFÉRENCES}

BAKER, M. (2000): “Towards a methodology for investigating the style of a literary translator", Target 12-2, pp. 241-266.

Cortanze, G. de. (1993): «Virginia Woolf, instants de vie», Magazine Littéraire 309, pp. 9596.

Forrester, V. (1993): «Qui a trahi Virginia Woolf?», Le Monde, 23 avril 1993, p. 30.

Munday, J. (1998): "A computer-assisted approach to the analysis of translation shifts", Meta 43-3, pp. 1-16.

Munday, J. (2002): "Systems in translation: a systemic model for descriptive translation studies", in Hermans, T. (ed.), Crosscultural Transgressions. Research Models in Translation Studies II: Historical and Ideological Issues, Manchester, St Jerome, pp. 76-92.

Reynier, C. (ed.) (1993): Études Britanniques Contemporaines $n^{\circ} 2$, Montpellier, Presses universitaires de Montpellier.

Roy, P. (1977): The Dual Voice: Free Indirect Speech an its Functioning in the Nineteenth-Century European Novel, Manchester, Manchester University Press and Rowan and Littlefield.

Woolf, V. (1927): To the Lighthouse, London, Penguin Popular Classics.

Woolf, V. (1939): Moments of Being, in Schulkind, J. (ed.), London, Chatto and Windus, Sussex University Press.

JAnzen, T. (ed.) (2005): Topics in Signed Language Interpreting: Theory and Practice, Philadelphia, John Benjamins, 362 p.

Russell, Malcolm, Stratiy, Demers, les grands noms de la scène canadienne en matière d'interprétation en langue des signes, conjuguent leurs visions à celles de Wilcox et Shaffer du Nouveau-Mexique et de Leeson de Dublin dans un ouvrage qui est d'ores et déjà une référence pour qui veut cerner les problématiques essentielles du domaine. Terry Janzen, directeur de Topics in Signed Language Interpreting, présente l'état le plus achevé des réflexions théoriques et pratiques dans le champ de l'interprétation impliquant au moins une langue des signes au Canada. Au Canada anglais, devrions-nous préciser. Le livre se divise en trois parties. La première se résume à une introduction et une présentation des auteurs. La deuxième aborde le champ de la théorie et la troisième, celui de la pratique.

Les deux premiers textes de la section théorique traitent du processus cognitif qui préside à l'interprétation. Wilcox et Shaffer reformulent une présentation archiconnue des différents modèles utilisés pour représenter le rôle de l'interprète en langue des signes: modèle mécaniste, biculturel, sociolinguistique... S'ensuit une critique du concept de neutralité en interprétation que nous pourrions dépasser par une approche constructiviste 
de la communication que les auteurs nomment «toolmakers paradigm». Ce modèle situe l'interprète comme un agent porteur de biais dont il peut se distancer et tendre vers la neutralité dans la mesure où il est conscient de son engagement dans la communication. Pour n'être pas nouveau, le propos des auteurs n'en demeure pas moins clair et bien argumenté.

Leeson prolonge la discussion au chapitre troisième en statuant également que l'interprète est un agent actif dans la communication et que la qualité de son travail dépend des stratégies qu'il déploie. En s'appuyant sur le modèle des efforts de Gile (1995), l'auteure présente les différentes stratégies permettant de maintenir l'équilibre des efforts alors que plusieurs d'entre elles, telles que l'ajout ou l'omission, sont traditionnellement vues comme étant des erreurs. Il est de première importance pour l'interprète, explique l'auteure, de faire les choix stratégiques les plus respectueux des normes éthiques de la profession tout en permettant à la rencontre de se dérouler avec succès. L'apport de Leeson est intéressant même si cette exploration du modèle de Gile appliquée à l'interprétation en langue des signes reste quelque peu superficielle et paraît tardive. Tardive, d'une part, si nous considérons l'écart qui sépare cette analyse de la publication de Basic Concepts and Models for Interpreter and Translator Training (Gile 1995) et, d'autre part, si nous établissons un parallèle avec ce "nouveau» regard et les pratiques dans la formation des interprètes en langue des signes au Québec. En effet, les travaux de Gile sont couramment utilisés dans l'enseignement de l'interprétation français/langue des signes québécoise (LSQ) depuis plus d'une dizaine d'années, les enseignants étant aidés en cela par la langue, car le premier article de Gile sur l'équilibre des efforts a trouvé une première forme française dans un article de Meta paru en 1985. Il est ici question, nous semble-t-il, de passerelles à bâtir entre les traductologues des langues orales et ceux des langues des signes tout comme entre les milieux anglophone et francophone.

Les chapitres 4 et 5 empruntent à la linguistique le cadre de référence pour traiter du rapport que l'interprète entretient avec les langues de travail. Janzen compare l'anglais et la langue des signes américaine (ASL) pour en faire ressortir les traits convergents et divergents. Il examine avec beaucoup d'acuité la problématique du bilinguisme des interprètes en langue des signes dont l'une des particularités est de travailler vers une langue seconde, l'ASL, rarement apprise dans l'enfance. Il avance plusieurs hypothèses pour expliquer l'influence de l'anglais sur la structure du message signé par les interprètes débutants dont les plus probantes sont la prééminence de la modalité audio-orale sur la modalité visuogestuelle et l'acquisition encore trop fragile de la langue des signes chez les interprètes. Au-delà de sa compréhension intuitive des langues de travail, l'interprète doit, comme l'auteur le fait voir, ancrer son interprétation sur le sens mais également la forme du message et, dans cette perspective, s'appuyer sur les ressources lexicale (word), morphosyntaxique (construction), discursive (texts) et contextuelle (context) pour réussir son interprétation.

Malcolm, au cinquième chapitre, s'intéresse également au phénomène de contact des langues dans le travail des interprètes, mais elle insiste, pour sa part, sur une forme d'interprétation particulière, voire unique, l'interprétation en langue des signes qui consiste à rendre une langue orale visuellement accessible par les moyens d'une langue des signes. On parle de translittération pour ce mode de réexpression du message dans une même langue, l'anglais dans les propos de Malcolm, d'une modalité audio-orale à une modalité visuo-gestuelle. Beaucoup moins prestigieuse que l'interprétation d'une langue orale vers une langue signée, la translittération n’en est pas moins une forme de médiation linguistique attendue de la part des interprètes lorsque ce besoin convient aux locuteurs requérant leurs services. Or, cet aspect du travail de l'interprète ne trouve aucun écho dans les programmes de formation des interprètes en langue des signes. On doit à Malcolm d'avoir, sans jamais aborder le problème dans ces termes, analysé les incidences du rapport diglossique entre langues signées et langues orales dans les modes d'interprétation effectifs auprès 
des communautés sourdes et le parti pris idéologique pour la défense et la reconnaissance des langues signées dans les programmes de formation d'interprètes. Une réserve pourtant, le titre, Contact sign, transliteration and interpretation in Canada, a de quoi irriter le lecteur qui évolue dans l'une des communautés francophones canadiennes (de l'Ontario ou du Québec, par exemple) où les langues de travail ne sont pas l'anglais et l'ASL mais bien le français et la LSQ. Quand bien même l'auteure annonce que les «implications for training and certification within Canada will be discussed», la discussion ne couvre jamais la réalité francophone.

Le sixième chapitre aborde le sujet de l'interprétation consécutive entre une langue orale et une langue signée. Il faut dire que la simultanée est pratiquement le mode d'interprétation par défaut chez les interprètes en langue des signes en raison de l'absence d'interférence entre les modalités audio-orale et visuo-gestuelle des langues de travail. Aussi, la consécutive n'a-t-elle jamais mérité l'attention qu'on lui porte dans l'interprétation entre deux langues orales. Toutefois, Russell met en lumière l'apport essentiel de ce mode d'interprétation qu'elle intègre à un modèle de décisions qui conduit l'interprète à choisir le mode d'interprétation simultané ou consécutif selon son analyse du contexte et de sa compréhension du message source dans toute sa complexité (registre, style...). Ses études portant sur des interprétations en contexte judiciaire démontrent que les erreurs d'interprétation augmentent en situation d'interprétation simultanée et que l'interprétation consécutive entre une langue orale et une langue signée reste une compétence professionnelle à développer dans ce champ de pratique.

Fermant la section théorique, l'éthique occupe le septième chapitre. À nouveau, certains modèles retenus pour guider les conduites professionnelles des interprètes en langue des signes font l'objet de commentaires. On s'étonne des tournures hypothétiques du style "While there may be numerous models by which interpreters define themselves, the description here will be restricted to the several most commonly used labels [...]» (p. 166) alors que le deuxième chapitre a clairement marqué le point et que ce tour de piste est pratiquement devenu un lieu commun des études en interprétation en langue des signes. Néanmoins, Janzen et Korpiniski livrent une lecture critique du code de déontologie mis de l'avant par l'Association of Visuel Language Interpreters of Canada (AVLIC) en faisant voir que la faiblesse de la prise en compte des contextes et des cultures invalide la pertinence de plusieurs préceptes du code. Les auteurs ajoutent que la communauté sourde et les pairs doivent également figurer parmi les acteurs dont il faut tenir compte du point du vue des conduites professionnelles en vue de se gagner la confiance du milieu. L'impossible neutralité et le pouvoir que peut exercer l'interprète font répéter aux auteurs qu'il importe d'agir dans le meilleur intérêt des personnes qui requièrent les services de l'interprète.

La section pratique couvre cinq chapitres. L’ensemble est assez éclectique, mais comporte, comme fil conducteur, la présentation de stratégies d'interprétation selon différentes perspectives: les stratégies de préparation et d'organisation de la tâche d'interprète (chapitre 8), les stratégies d'interprétation au regard des enjeux psycho-sociologiques avec en leur centre les relations avec la communauté sourde du Canada anglais (chapitre 9), les stratégies d'interprétation visant à résoudre des problèmes terminologiques découlant des variations linguistiques parmi les locuteurs des langues des signes (chapitre 10), des stratégies d'intervention professionnelle en contexte d'interprétation scolaire (chapitre 11) et, finalement, l'interprétation entre deux langues des signes.

Le caractère appliqué des propositions de cette partie présente une grande valeur. Le lecteur, surtout s'il débute en interprétation, détient l'occasion de se construire un vademecum des pratiques à faire et à ne pas faire. Avec Demers, il saura formaliser sa préparation et agir de façon systématique pour assurer le succès d'une séance d'interprétation. 
Dans l'exercice de ses fonctions, il portera une attention particulière aux dimensions pragmatique, culturelle et linguistique de sa pratique que Stratiy rappelle non sans pertinence puisque ces éléments fondamentaux dans la formation des interprètes sont repris ici selon le point vue de la communauté sourde. Malheureusement, une rupture marque le passage au texte de Leeson. Les variations linguistiques sexo-spécifiques et générationnelles ne constituent pas un phénomène étranger aux Sourds du Canada, mais cette étude sur les locuteurs de la langue des signes irlandaise, malgré un volet appliqué, détonne. Alors qu'on nous entretenait de considérations très pointues sur la manière d'interpréter tel ou tel mot de l'anglais à l'ASL, Leeson introduit son propos par une mise en contexte très large de l'interprétation en langue des signes irlandaise et des particularités linguistiques de cette langue avant de réserver dix des quarante pages de son article, le plus long du volume, aux stratégies d'interprétation en regard des variations linguistiques en Irlande et lors des rencontres internationales de la communauté européenne. Le texte, malgré ses qualités, donne l'impression d'avoir été plaqué au beau milieu d'une discussion qui, néanmoins, reprend son cours. En effet, la difficulté de circonscrire le rôle professionnel de l'interprète en milieu scolaire occupe le chapitre suivant et nous replonge dans des considérations pratiques à l'égard de situations ancrées dans le contexte nord-américain. On doit aux auteurs d'explorer finement les enjeux éthiques que pose la pratique de l'interprétation sur le long terme et dans un milieu institutionnel où les frontières de rôles (interprète, éducateur, tuteur...) sont parfois poreuses. L'apport spécifique des auteurs, sans être totalement novateur, réside peut-être dans l'analyse approfondie des questions de dominance et d'exercice du pouvoir qui affectent la relation interprète-client sourd, le premier faisant partie de la majorité entendante et le second, d'une communauté minoritaire. Le dernier chapitre est consacré à la description détaillée de l'interprétation entre deux langues des signes, une pratique émergente réalisée principalement par des interprètes sourds et dont le degré de formalisation des pratiques gagne du terrain. Boudreault offre une superbe synthèse qui embrasse un ensemble de questionnements fort pertinents (relation des interprètes sourds avec la communauté sourde, formation des interprètes sourds, application du code de déontologie...).

Avec celui de Demers, qui ouvre la section pratique, l'article de Boudreault présente une contribution originale qui dépasse la recension critique de théories ou d'approches relativement connues des lecteurs avertis. En effet, s'il a fréquenté les congrès de l'Association of Visual Language Interpreters of Canada (AVLIC) et autres colloques en interprétation en langue des signes ces dernières années, le lecteur reconnaît les approches et les préoccupations qui animent les auteurs. Les perspectives d'un Janzen et plus encore d'une Russell sur l'interprétation consécutive dans les cours de justice font en effet l'objet d'écrits et de conférences depuis plusieurs années.

Point de détail, affirmer, comme c'est le cas en introduction, que ce recueil reflète la sensibilité canadienne et les récents développements significatifs dans le domaine de l'interprétation en langue des signes au Canada, y compris les paires de langues ASL-anglais, ASL-LSQ et LSQ-français (p. 3-4), c'est tourner les coins rond, pour reprendre une expression bien québécoise. En effet, le lecteur québécois, justement, membre de la communauté sourde en milieu francophone ou interprète LSQ-français, ne retrouve pas nécessairement son reflet dans ce volume. Par exemple, certaines perspectives nouvelles ne le sont pas pour le milieu québécois, de même, les références à LA perspective de la communauté sourde mériteraient d'être nuancées, car il y a deux communautés sourdes au Canada et les différences entre les Sourds québécois et les Sourds du Canada anglais sont bien réelles. Rien de dommageable, néanmoins, il suffit de garder en mémoire qu'on ne peut si facilement englober toute la réalité canadienne. Une précaution dans ce sens améliorerait le texte en plusieurs endroits. 
Somme toute, même s'il y a peu d'apports nouveaux, l'ensemble est bien tenu, l'effort de cohésion et les notes de renvoi entres les articles sont appréciés, ces regards sur l'interprétation des langues de signes ont beaucoup d'intérêt. Nous ne regrettons qu'une seule chose, le coût élevé du volume qui le rend sûrement peu accessible en dehors des bibliothèques universitaires.

Danielle-Claude Bélanger

Université du Québec à Montréal, Montréal, Canada

\section{RÉFÉRENCES}

Giles, D. (1995): Basic Concepts and Models for Interpreter and Translator Training, Amsterdam/Philadelphia, John Benjamins.

Delisle, J. et J. Woodsworth (dir.) (2007): Les traducteurs dans l'histoire, Ottawa, Presses de l'Université d'Ottawa, $2^{e}$ édition revue et corrigée, xxiii-393 p.

Ce livre était à l'origine une gageure, mais il est heureux de voir aujourd'hui, une dizaine d'années plus tard, une deuxième édition, ainsi que quelques traductions dans diverses langues. Ce livre est en fait une esquisse - pouvait-on faire davantage en si peu de pages? C'est un condensé qui illustre les expériences antérieures et les résultats obtenus par des pionniers traducteurs. C'est en fait un livre de créations et de résultats que nous pouvons considérer comme prototypiques en ce sens qu'ils ne forment pas un tout mais seulement des exemples que l'on peut (doit?) multiplier et adapter en changeant de langue de référence ou de pays puisqu'il y aura beaucoup en commun mais également une constante individuelle à mettre en relief. Cela montre bien que l'ouvrage répond encore à un besoin et que l'entreprise est encore des plus méritoires. Rappelons-le, même si on le sait parfaitement, que résumer en si peu de pages l'histoire d'un certain nombre de traducteurs, et donc de la traduction, et par conséquent synthétiser l'ensemble de la civilisation mondiale des origines à nos jours, tant pour les sciences et la technologie que pour l'histoire, la littérature et les influences politiques et religieuses, est une entreprise sinon impossible du moins périlleuse, car il faut en peu de lignes dire l'essentiel et faire percevoir la trame de l'ensemble. Tout devient forcément élément de choix et bien évidemment interprétation et mise en relief de quelques aspects fondamentaux pour les orientations retenues. La critique devient aisée, car le choix des secteurs retenus peut être considéré comme partiel et surtout partial, et lié aux intérêts directs des auteurs. Mais il faut quand même souligner que l'essentiel pour ces secteurs est présent et que le choix n'est en quelque sorte qu'une mise en bouche qui laisse deviner l'attrait pour la suite et tout l'intérêt des développements qui s'annoncent et des compléments de lecture possibles. Les exemples donnés sont donc souvent à considérer comme des cas d'espèces hautement généralisables.

L'ouvrage suit un ordre logique et répartit la matière en neuf chapitres, chacun est suivi de références pour des lectures complémentaires. Ainsi au chapitre premier «Les traducteurs, inventeurs d'alphabets" font suite les chapitres où on les reconnaît comme «bâtisseurs de langues nationales, artisans de littératures, diffuseurs de connaissances, acteurs sur la scène du pouvoir, propagateurs de religions, importateurs de valeurs, consommateurs mais aussi compilateurs de dictionnaires terminologiques ». Le dernier chapitre s'attache au rôle des «interprètes témoins privilégiés de l'histoire». L'ouvrage se termine par deux annexes (légendes des illustrations; coauteurs), les «Références» et une «Bibliographie complémentaire - Domaines anglais et français (1995-2006)» et un «Index» des auteurs cités. 